CERN-TH.7336/94

\title{
RESUMMATION OF PERTURBATION SERIES IN NON-EQUILIBRIUM SCALAR FIELD THEORY
}

\author{
T. Altherr虫 \\ Theory Division, CERN, CH-1211 Geneva 23, Switzerland
}

\begin{abstract}
The general behaviour of perturbation series in non-equilibrium scalar field theory is analysed in some detail, with a particular emphasis on the "pathological terms", generated by multiple products of $\delta$-functions. Using an intuitive regularization method, it is shown that these terms give large contributions at all orders, even when considering small deviations from equilibrium. Fortunately, these terms can also be resummed and I give the general expressions for the resummed propagators in non-equilibrium scalar field theory, regardless of the size of deviations from equilibrium.
\end{abstract}

Submitted to Physics Letters B

CERN-TH.7336/94

June 94

\footnotetext{
${ }^{1}$ On leave of absence from L.A.P.P., BP110, F-74941 Annecy-le-Vieux Cedex, France.
} 


\section{Introduction}

In a previous work [1], Seibert and the present author showed that the standard theoretical framework of non-equilibrium quantum field theory is plagued with serious difficulties when perturbation series is considered. The problem originates from the non-cancellation of the "pathological terms" [2] that are associated to multiple products of $\delta$-functions. In [1], it has been proved that these terms do not cancel unless the particle distributions are those for a system in thermal and chemical equilibrium.

This paper is devoted to the discussion of a possible solution to this problem. In particular, the behaviour of the perturbation series, that is in terms of the coupling constant, is scrutinized in the light of the presence of these pathologies. First needed is a regularization scheme, in order to deal with the mathematically ill-defined pathologies. Since the problem originates from the infinitesimal $i \epsilon$ prescription in the free propagator, a rather natural way to perform this regularization is to introduce a finite width, or damping, in the propagators.

Throughout the paper, the $2 \times 2$ Keldysh matrix structure [3] is used as a toy-model of a non-equilibrium quantum field system,

$$
\begin{aligned}
& \left(\begin{array}{cc}
D_{11}(K) & D_{12}(K) \\
D_{21}(K) & D_{22}(K)
\end{array}\right)= \\
& \left(\begin{array}{cc}
\Delta_{R}(K)\left(1+n\left(k_{0}\right)\right)+\Delta_{A}(K) n\left(k_{0}\right) & \left(1+n\left(k_{0}\right)\right)\left(\Delta_{R}(K)+\Delta_{A}(K)\right) \\
n\left(k_{0}\right)\left(\Delta_{R}(K)+\Delta_{A}(K)\right) & \Delta_{R}(K) n\left(k_{0}\right)+\Delta_{A}(K)\left(1+n\left(k_{0}\right)\right)
\end{array}\right) 1,
\end{aligned}
$$

with the Retarded/Advanced propagators defined as

$$
\Delta_{R(A)}(K)=\frac{+(-) i}{K^{2}-m^{2}+(-) i \gamma k_{0}} .
$$


The width $\gamma$ is an arbitrary finite function of $K$. The distribution $n\left(k_{0}\right)$ is defined so that

$$
n\left(k_{0}\right)=-1-n\left(-k_{0}\right)=\mathbf{n}(\mathbf{k}),
$$

where $\mathbf{n}(\mathbf{k})$ represents the non-equilibrium distribution of the quanta, namely

$$
\operatorname{Tr}\left[\rho a^{\dagger}(\mathbf{k}) a(\mathbf{p})\right]=\mathbf{n}(\mathbf{k}) \delta^{3}(\mathbf{k}-\mathbf{p})
$$

where $\rho$ is an arbitrary density operator. It is assumed that the system evolves sufficiently slowly in time and space so that the Fourier transform, for short space-time separations, has the simple expression shown in eq. (1).

By introducing this finite width in the bare propagators, the perturbation series now becomes mathematically well-defined: pathologies associated with multiple products of $\delta$-functions are regularized. In principle, this width, which is introduced heuristically "by hand", can be calculated by using perturbation theory. I shall discuss this point in more detail later on.

The question is now: How do the pathological terms change the behaviour of the perturbation series in terms of the coupling constant $g$ ?

\section{$2 g^{2} \phi^{4}$ theory}

From now on, I consider the massless case. The simplest thing to compute in $\left(g^{2} / 4 !\right) \phi^{4}$ theory is the tadpole diagram (in $n=4-2 \epsilon$ dimensions)

$$
\operatorname{Re} \Sigma=\frac{1}{2} g^{2} \int \frac{d^{n} K}{(2 \pi)^{n}}\left(1+2 n\left(k_{0}\right)\right) \frac{\gamma k_{0}}{K^{4}+\gamma^{2} k_{0}^{2}} .
$$

As usual, the ultraviolet singularities are present in the vacuum part only, provided the distribution $n\left(k_{0}\right)$ drops sufficiently fast when $k_{0} \rightarrow \infty$, as in 
the equilibrium case. For the matter part (denoted by $\delta \Sigma$ in the following), I obtain, after a Cauchy integration:

$$
\operatorname{Re}(\delta \Sigma)=\frac{g^{2}}{8 \pi^{2}} \int_{0}^{\infty} d k_{0} k_{0} n\left(k_{0}\right)\left(\sqrt{1+i \frac{\gamma}{k_{0}}}+\sqrt{1-i \frac{\gamma}{k_{0}}}\right),
$$

where I have assumed, for the sake of simplification in the notation, that $\gamma$ depends only on $k_{0}$. One recovers the well-known thermal mass, $\delta m^{2}=$ $\left(g^{2} / 24\right) T^{2}$, when $\gamma \rightarrow 0$ and when $n\left(k_{0}\right)$ is the Bose-Einstein distribution [四]. At one loop, the imaginary part of $\Sigma$ vanishes, as in the case of zero width.

Hence, eq. (6) provides the complete non-equilibrium generalization of the one loop thermal mass at equilibrium, regardless of the particle number distribution $n\left(k_{0}\right)$. In the case of a small damping one has

$$
\delta m^{2}=\frac{g^{2}}{4 \pi^{2}} \int_{0}^{\infty} d k_{0} k_{0} n\left(k_{0}\right)
$$

An inspection of the two loop diagrams does not reveal any serious infrared problem. For the first topology, shown in fig. 1a, terms that are associated with potential pathologies $\left(\delta^{2}\left(K^{2}\right)\right.$ terms) cancel in the limit of vanishing $\gamma$ [1]. Therefore, provided $n\left(k_{0}\right)$ is no more singular than the Bose-Einstein distribution, the infrared problems are not worst than at equilibrium. For instance, for a system that is close to equilibrium, the two loop diagrams contribute at $O\left(g^{3} T^{2}\right)$ to the thermal mass 4 .

The same is true for the second topology, shown in fig. 1b, where no pathologies are present. This diagram has an imaginary part, which also gives the width:

$$
\gamma(K)=\frac{i}{2 k_{0}}\left(\Sigma_{12}(K)-\Sigma_{21}(K)\right)
$$


For small deviations from equilibrium, $\gamma$ is just the usual equilibrium damping rate in $g^{2} \phi^{4}$ theory, that is

$$
\gamma_{\text {hard }}=O\left(g^{4} T\right),
$$

times eventually some $\ln 1 / g$ that arises from some logarithmic infrared singularities (I do not discuss these minor corrections here). The subscript hard refers to the case of a hard external momentum $(K \sim T)$. The soft $(K \sim g T)$ damping rate is easily calculable and is given by

$$
\gamma_{\text {soft }}=\frac{1}{32 \sqrt{6} \pi^{3}} g^{3} \ln \frac{1}{g} T,
$$

to leading order in $\ln 1 / \mathrm{g}$. As motivated from the beginning, the dampings, either soft or hard, eventually provide a way to regularize the pathological terms. However, there remains to perform the resummation of the damping effects that lead to the propagator (2) (which is only a useful assumption for the time being).

Potential problems arise at the next order, that is at the three loop order, which makes the analysis somewhat involved. As at the two loop order, the repeated tadpole insertions do not lead to any pathological terms. So the diagram shown in fig. 2a is no more singular than at equilibrium, and is of $O\left(g^{3} T^{2}\right)$, again for small deviations from equilibrium [4]. On the other hand, pathological terms appear in the diagram shown in fig. 2b. Upon using some useful relations between the different components of the self-energy insertion $\left.\Sigma^{1 b} \llbracket 1\right]$, one may write

$$
\begin{aligned}
-i \Sigma^{2 b}= & -i g^{2} \int \frac{d^{n} K}{(2 \pi)^{n}}\left\{-i \operatorname{Re} \Sigma^{1 b}(K)\left[D_{11}^{2}(K)-D_{12}(K) D_{21}(K)\right]\right. \\
& -i \Sigma_{12}^{1 b}(K)\left[-\frac{1}{2}\left(D_{11}^{2}(K)+D_{12}(K) D_{21}(K)\right)+D_{11}(K) D_{21}(K)\right]
\end{aligned}
$$




$$
-i \Sigma_{21}^{1 b}(K)\left[-\frac{1}{2}\left(D_{11}^{2}(K)+D_{12}(K) D_{21}(K)\right)+D_{11}(K) D_{12}(K)(1\}\right)
$$

In terms of Retarded/Advanced propagators:

$$
\begin{aligned}
\Sigma^{2 b}= & g^{2} \int \frac{d^{n} K}{(2 \pi)^{n}}\left\{-i \operatorname{Re} \Sigma^{1 b}(K)\left[\left(1+n\left(k_{0}\right)\right) \Delta_{R}^{2}(K)-n\left(k_{0}\right) \Delta_{A}^{2}(K)\right]\right. \\
& +i \frac{1}{2} \Sigma_{12}^{1 b}(K)\left[\left(1+n\left(k_{0}\right)\right) \Delta_{R}^{2}(K)+n\left(k_{0}\right) \Delta_{A}^{2}(K)+2 n\left(k_{0}\right) \Delta_{R}(K) \Delta_{A}(K)\right] \\
& -i \frac{1}{2} \Sigma_{21}^{1 b}(K)\left[\left(1+n\left(k_{0}\right)\right) \Delta_{R}^{2}(K)+n\left(k_{0}\right) \Delta_{A}^{2}(K)+2\left(1+n\left(k_{0}\right)\right) \Delta_{R}(K) \Delta_{A}(K)(1 \mathrm{f})\right.
\end{aligned}
$$

The pathological terms are thus

$$
\begin{aligned}
\Sigma^{2 b}= & g^{2} \int \frac{d^{n} K}{(2 \pi)^{n}} \Delta_{R}(K) \Delta_{A}(K)\left[-i \Sigma_{12}^{1 b}(K) n\left(k_{0}\right)+i \Sigma_{21}^{1 b}(K)\left(1+n\left(k_{0}\right)\right)\right] \\
& + \text { regular terms. }
\end{aligned}
$$

Let me now define a function $f$ such that

$$
-i \Sigma_{12}^{1 b}(K) n\left(k_{0}\right)+i \Sigma_{21}^{1 b}(K)\left(1+n\left(k_{0}\right)\right)=g^{4} f\left(k_{0}\right) \delta n\left(k_{0}\right),
$$

where $\delta n\left(k_{0}\right)=n\left(k_{0}\right)-n_{B}\left(k_{0}\right)$ represents the non-equilibrium deviations. The pathological contribution to the three loop order reads

$$
\Sigma^{2 b}=\frac{g^{6}}{16 \pi^{2}} \int_{-\infty}^{+\infty} d k_{0} f\left(k_{0}\right) \delta n\left(k_{0}\right) \frac{1}{\gamma}\left[\sqrt{1+i \frac{\gamma}{k_{0}}}+\sqrt{1-i \frac{\gamma}{k_{0}}}\right],
$$

which shows that, under the assumption that $f\left(k_{0}\right)$ and $\delta n\left(k_{0}\right)$ do not have a singular infrared behaviour,

$$
\Sigma^{2 b}=O\left(g^{6} \frac{\delta n(\kappa) \kappa^{3}}{\gamma}\right)=O\left(g^{2} \delta n(\kappa) \frac{\kappa^{3}}{T}\right),
$$

where $\kappa$ is the momentum scale that dominates the integral in eq. (15) (it is associated to the fluctuations). These simple assumptions about the behaviour of the fluctuation terms are just made to illustrate the possible breakdown of perturbation series in the presence of the pathological terms. Let me 
further assume that the scale $\kappa$ does not differ too much from the temperature T. Now, if

$$
\delta n(\kappa)=O\left(g^{2}\right)
$$

the pathological term is larger than the regular terms in eq. (12) (that can be shown to be of $O\left(g^{5}\right)$ ). But it is still smaller by $O(g)$ than the tadpole insertions diagram (fig. 3), which is the dominant infrared diagram. This indicates that it might be possible to get a control over the perturbation series in that case.

However, the above contribution is also of the same order, $O\left(g^{4}\right)$, as the non-equilibrium corrections to the first-order result (see eq. (7)). Therefore, nothing more than the equilibrium limit can be learned from eq. (17) without calculating these terms.

Not surprisingly, it also turns out that the higher-order self-energy insertions contribute at the same order for the pathological term. After some algebra, one can show that (see fig. 3):

$$
\begin{aligned}
\Sigma^{(N)}= & g^{2} \int \frac{d^{n} K}{(2 \pi)^{n}}\left\{(1+n) \Delta_{R}\left(-i \Sigma \Delta_{R}\right)^{N}+n \Delta_{A}\left(+i \Sigma^{*} \Delta_{A}\right)^{N}\right. \\
& \left.-i \Delta_{R} \Delta_{A}\left(n \Sigma_{12}-(1+n) \Sigma_{21}\right) \sum_{n=0}^{N-1}\left(-i \Sigma \Delta_{R}\right)^{N-1-n}\left(+i \Sigma^{*} \Delta_{A}\right)^{n}(\} 8\right)
\end{aligned}
$$

In order to simplify the notation, I have removed the $K$-dependence in all the quantities: $n\left(k_{0}\right), \Delta(K)$ and $\Sigma(K)$. One important remark must be added: the self-energy $\Sigma$ that enters in the above expression must be a 2 point function, it does not include the tadpole.

The usual way of dealing with this kind of formula is to use the "massderivative formula" [5], which can be replaced by the "width-derivative formula" in the same fashion. Equation (18) shows one important feature: the 
pathological terms are always linear in $\delta n$. A simple estimate leads to

$$
\Sigma^{(N)}=O\left(g^{2} \delta n(\kappa)\left(\frac{T}{\kappa}\right)^{N} \frac{\kappa^{4}}{T^{2}}\right),
$$

where I have supposed that the $k_{0}$-integral is infrared safe (which is most probably not the case, making the behaviour shown above rather "optimistic"). Therefore, regardless of the respective size of the pathological terms versus the regular ones, it is clear that every repeated self-energy insertions must be taken into account, as it can contribute to the same order in the coupling constant.

The resummation of the non-pathological terms in eq. (18) is a wellknown exercise 四. The nice surprise is that the pathological terms can also be resummed, leading to

$$
\begin{aligned}
\sum_{N=0}^{\infty} \Sigma^{(N)}= & g^{2} \int \frac{d^{n} K}{(2 \pi)^{n}}\left\{(1+n) \frac{\Delta_{R}}{1+i \Sigma \Delta_{R}}+n \frac{\Delta_{A}}{1-i \Sigma^{*} \Delta_{A}}\right. \\
& \left.-i\left(n \Sigma_{12}-(1+n) \Sigma_{21}\right) \frac{\Delta_{R}}{1+i \Sigma \Delta_{R}} \frac{\Delta_{A}}{1-i \Sigma^{*} \Delta_{A}}\right\} .
\end{aligned}
$$

This is the central result. Besides the usual first terms that are similar to the equilibrium resummation, the last term contains all the non-equilibrium diseases that are cured in this rather elegant way. One can see that this term behaves as eq. (15),

$$
\Sigma_{\text {pathology }}=O\left(g^{2} \delta n\right) .
$$

In principle, with this resummation, one is not restricted to the study of systems that are close to equilibrium. For $\delta n>O(1)$, the pathological term becomes the leading contribution to the mass shift, and most probably to all other quantities.

Finally, I give the expression for the resummed propagator, which can be 
read off from eq. (20):

${ }^{*} D_{11}(K)=\left(1+n\left(k_{0}\right)\right){ }^{*} \Delta_{R}(K)+n\left(k_{0}\right){ }^{*} \Delta_{A}(K)+2 k_{0} \Delta n\left(k_{0}\right){ }^{*} \Delta_{R}(K){ }^{*} \Delta_{A}(K)$,

where the ${ }^{*}$ refers to the resummed quantities. Note that the following relation has been used [6]

$$
\begin{aligned}
2 k_{0} \Delta n\left(k_{0}\right) & =-i\left(n\left(k_{0}\right) \Sigma_{12}(K)-\left(1+n\left(k_{0}\right)\right) \Sigma_{21}(K)\right) \\
& =2 k_{0}\left(\frac{d n\left(k_{0}\right)}{d t}+\mathbf{v} \cdot \nabla n\left(k_{0}\right)\right)
\end{aligned}
$$

which is nothing but the quantum-field Boltzmann equation [3]. For completeness, I also list the resummed expression for the other matrix components

${ }^{*} D_{22}(K)=\left({ }^{*} D_{11}(K)\right)^{*}$

${ }^{*} D_{12}(K)=\left(1+n\left(k_{0}\right)\right)\left({ }^{*} \Delta_{R}(K)+{ }^{*} \Delta_{A}(K)\right)+2 k_{0} \Delta n\left(k_{0}\right){ }^{*} \Delta_{R}(K){ }^{*} \Delta_{A}(K)$

${ }^{*} D_{21}(K)=n\left(k_{0}\right)\left({ }^{*} \Delta_{R}(K)+{ }^{*} \Delta_{A}(K)\right)+2 k_{0} \Delta n\left(k_{0}\right){ }^{*} \Delta_{R}(K){ }^{*} \Delta_{A}(K)$.

\section{$3 g \phi^{3}$ theory}

For completeness, I also analyse the behaviour of perturbation series in $(g / 3 !) \phi^{3}$ theory (in 6 space-time dimensions). It is evident that the situation can be different, as the repeated self-energy insertions on the same line contain some pathological terms and is at the same time the leading infrared series in the equilibrium limit.

Let me begin with the real part of the one loop self-energy. As the interest here is to make a possible generalization to the case of gauge theories, I 
discard the tadpole diagram from the discussion [7]. One has,

$$
\operatorname{Re} \Sigma(P)=\frac{1}{2} g^{2} \int \frac{d^{n} K}{(2 \pi)^{n}} 2 \operatorname{Re} D_{11}(K) \operatorname{Im} D_{11}(P-K) .
$$

In order to simplify the calculations, I use the "Hard Thermal Loop" approximation, that is I consider small external momenta and I suppose that the dominant contribution to the integral is such that $P \ll K$. Using the same techniques as in the $g^{2} \phi^{4}$ case, I easily obtain

$$
\operatorname{Re}(\delta \Sigma(P))=-\frac{g^{2}}{64 \pi^{3}} \int_{0}^{\infty} d k_{0} k_{0} n\left(k_{0}\right)\left(\sqrt{1+i \frac{\gamma}{k_{0}}}+\sqrt{1-i \frac{\gamma}{k_{0}}}\right)
$$

Again, one recovers the well-known thermal mass, $\delta m^{2}=-g^{2} /(384 \pi) T^{2}$, when $\gamma \rightarrow 0$ and when $n\left(k_{0}\right)$ is the Bose-Einstein distribution [7].

Now, pathological terms arise already at the two loop level. This is easily seen in the diagram shown in Fig. 4, which gives

$$
\begin{aligned}
\Sigma_{11}^{4}(P)= & -i g^{4} \int \frac{d^{n} K}{(2 \pi)^{n}} \Delta_{R}(K) \Delta_{A}(K) \\
& \times\left[-i \Sigma_{12}^{(1)}(K) n\left(k_{0}\right)+i \Sigma_{21}^{(1)}(K)\left(1+n\left(k_{0}\right)\right)\right] D_{11}(P-K) \\
& + \text { regular terms. }
\end{aligned}
$$

Using the same assumptions as in the previous case (eq. (14)), I obtain (in HTL approximation):

$$
\operatorname{Re} \Sigma_{11}^{4}=-\frac{g^{4}}{64 \pi^{3}} \int_{-\infty}^{+\infty} d k_{0} f\left(k_{0}\right) \delta n\left(k_{0}\right) \frac{1}{\gamma}\left[\sqrt{1+i \frac{\gamma}{k_{0}}}+\sqrt{1-i \frac{\gamma}{k_{0}}}\right]
$$

For small deviations from equilibrium, the damping is of order $g^{4} T$, and therefore

$$
\operatorname{Re} \Sigma_{11}^{4}=O(\delta n)
$$

Although the breakdown of perturbation series seems worse than in the previous case, this is not so. The reason for this is the regularization scheme 
that is not adequate here. Indeed, the resummed propagator that includes all the pathological terms is the same as in the previous case (see eq. (22)). But there is now a non-vanishing contribution at $O\left(g^{2}\right)$ for the 2-point Green's function (this is just the thermal mass, eq. (26)), which acts as an infrared regulator. Therefore the above contribution is much smaller

$$
\operatorname{Re} \Sigma_{11}^{4}=O\left(g^{2} \delta n\right)
$$

that is the same as in the $g^{2} \phi^{4}$ case. Hence, I end up with the same conclusions. Pathological terms will be the leading effects whenever considering deviations from equilibrium such that $\delta n>O(1)$.

\section{Conclusion and outlook}

The behaviour of perturbation series in the presence of pathologies, associated to multiple products of $\delta$-functions, has been analysed in some detail - Although the regularization method used here is very heuristic, I believe that it illustrates well the fact that, even for small deviations from equilibrium, the perturbation series break down. Anyway, the central result of this work is to show that these large contributions can be resummed. The resulting theory is free of pathologies and set up a coherent framework for the study of non-equilibrium systems, even in the case of large deviations from equilibrium.

Of course, depending on the distribution $n\left(k_{0}\right)$, some additionnal infrared problems may show up, but this needs a case-by-case study.

As usual in thermal field theory, the results that have been found here in scalar theory should easily translate to the case of gauge theories. There 
remains to be seen whether or not gauge-invariance problems show up when using a resummed propagator such as eq. (22).

\section{Acknowledgements}

I wish to thank F. Gelis, R. Kobes, T. del Rio Gaztelurrutia and D. Seibert for discussions. 


\section{References}

[1] T. Altherr and D. Seibert, preprint CERN-TH.7271/94, to appear in Phys. Lett. B.

[2] N. P. Landsman and Ch. G. van Weert, Phys. Rep. 145 (1987) 141.

[3] L. V. Keldysh, Sov. Phys. 20 (1964) 1018; K. C. Chou et al., Phys. Rep. 118 (1985) 1; J. Rammer and H. Smith, Rev. Mod. Phys. 58 (1986) 323; A. J. Niemi, Phys. Lett. B203 (1988) 425; E. Calzetta and B. L. Hu, Phys. Rev. D37 (1988) 2878.

[4] H. Matsumoto, I. Ojima and H. Umezawa, Ann. Phys. (NY) 152 (1984) 348; T. Altherr, Phys. Lett. B238 (1990) 360; R. Parwani, Phys. Rev. D48 (1993) 3852.

[5] Y. Fujimoto and R. Grigjanis, Z. Phys. C28 (1985) 395.

[6] H. A. Weldon, Phys. Rev. D28 (1983) 2007.

[7] T. Altherr, T. Grandou and R. D. Pisarski, Phys. Lett. B271 (1991) 183. 


\section{Figure captions}

Fig. 1 The two loop order contribution to the self-energy in $g^{2} \phi^{4}$.

Fig. 2 The leading infrared three loop order contribution to the self-energy in $g^{2} \phi^{4}$.

Fig. $3 N$ self-energy insertions on the tadpole loop.

Fig. 4 A two loop order contribution to the self-energy in $g \phi^{3}$. 


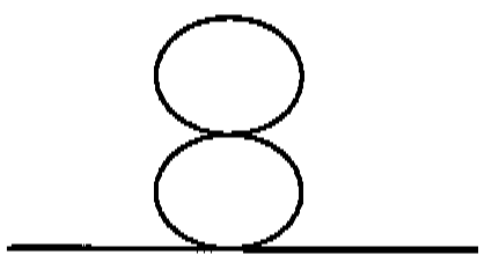

a)

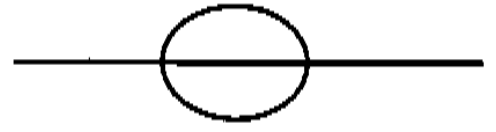

b)

Fig. 1
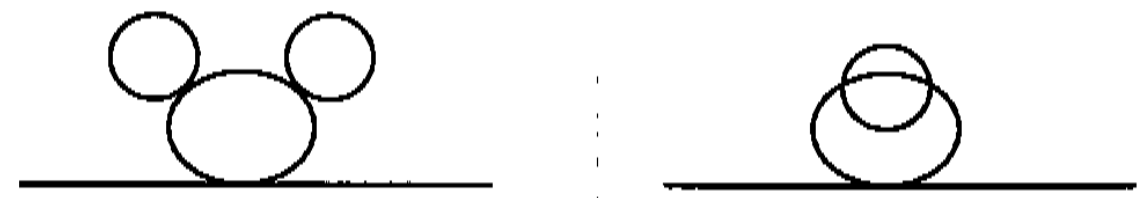

a)

Fig. 2

b)

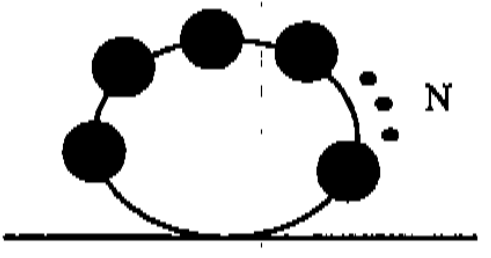

Fig. 3

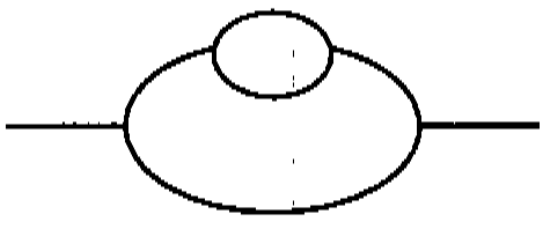

Fig. 4 
This figure "fig1-1.png" is available in "png" format from: http://arxiv.org/ps/hep-ph/9407249v1 